\title{
The construction of the electromagnetic current operator for the process of decay of the rho-meson in the different form of the Poincare-invariant quantum mechanics
}

\author{
Roman Polezhaev* \\ Samara State University \\ E-mail: polezaev@list.ru
}

\section{Krutov Alexander}

Samara State University

E-mail: krutov@samsu.ru

\begin{abstract}
This work is devoted to the description of radiation decays $V \rightarrow P \gamma$ in the framework of Poincareinvariant quantum mechanics (PIKM). A procedure developed for constructing the operator of the electromagnetic current of the transition with different values of the total angular momentum with respect to the conditions of the Lorentz covariance and conservation. As the figures of this approach in a modified relativistic impulse approximation held description of the radiative transition in the instant form PIKM.
\end{abstract}

The XXI International Workshop High Energy Physics and Quantum Field Theory,

June 23-June 30, 2013

Saint Petersburg Area, Russia

${ }^{*}$ Speaker. 


\section{Introduction}

Recently there have been several experiments study of radiative decays of vector mesons. So collaborations NA 60 [1]-[3] and KLOE-2 [4], were measured transition form factors in the reaction $\omega \rightarrow \pi \gamma, \phi \rightarrow \eta \gamma$ and evaluated the relevant constant decay. In [5] in the radiative decay $\rho \rightarrow \pi \gamma$ are studied deposits pions in section decays of vector mesons and received the necessary width radiative decay.

The theoretical description of the hadron structure and dynamics of their interaction is used, as a rule, the so-called quantum chromodynamics (QCD), which gives the quantitative description processes only at high energies. Therefore, for consideration related hadronic states have to use different phenomenological models, for example [6]-[9]. One of the the most frequently used is the composite quark model, however, in a theoretical description of the processes within the the model arises the problem of constructing the operator of electromagnetic current transition given the conditions of the Lorentz covariance and conservation. Natural for this model, impulse approximation violates these conditions. For example, in the framework of quantum field theory are obtained analytical expressions for the transition form factors of mesons theoretical curves of [10]-[11], analysis of which conclusion can be made about the difference results for form factors in the different approaches in describing one and the same process. Perhaps the reason for such a difference is the difference in the the procedure of constructing the matrix element of the electromagnetic current. Thus, the exploration of methodologies for the description of bound states is not lose its relevance in the present.

In this paper, in describing the processes $V \rightarrow P \gamma$ approach is used, the PIKM, implemented in three main forms: a point, instant form and dynamics on the light front, [12].

Proposed in this study differs to build procedure the operator of the electromagnetic current [13]. This procedure allows to select from the matrix element of the operator in any tensor dimension of the matrix elements (form factors), which are the invariants of the transformation of the Poincare group. It is worth noting that the construction of an operator current is carried in a modified relativistic momentum approximation (MIP), which does not lead to a breakdown of Lorentz - covariance and conservation. The build procedure the matrix element of the current transition for the case of a diagonal on the full angular moment was successfully used in the study of electromagnetic structure of peony and deuteron, including areas large momentum transfer [14]-[16].

The presentation of this method is in the instant form of dynamics for example, the description of radiation transition $\rho \rightarrow \pi \gamma$. For the first time when building the matrix element of the electromagnetic current transition for this process in the MIP used non-digional parameterization [17].

\section{Construction of the operator of the electromagnetic current of the transition to a system of noninteracting particles}

In this paper, we consider the electromagnetic current system of two noninteracting particles. State vector of the one of a free particle in the instant form PIKM can be represented as follows:

$$
|\vec{p}, M, j, m\rangle \text {. }
$$


In accordance with the General principles of quantum mechanics vector the status of two free particles with momenta $\vec{p}_{1}, \vec{p}_{2}$ and projections of spins $m_{1}, m_{2}$ on the axis of quantization in their systems of peace in the instant form of dynamics is constructed as a direct the product of the state vectors of individual particles:

$$
\left|\vec{p}_{1}, m_{1} ; \vec{p}_{2}, m_{2}\right\rangle=\left|\vec{p}_{1}, m_{1}\right\rangle \otimes\left|\vec{p}_{2}, m_{2}\right\rangle
$$

For the state vectors of (2.2) adopt the normalization:

$$
\begin{gathered}
\left\langle\vec{p}_{1}, m \mid \vec{p}_{1}{ }^{\prime}, m^{\prime}\right\rangle=2 p_{0} \delta\left(\vec{p}_{1}-\vec{p}_{1}{ }^{\prime}\right) \delta_{m m^{\prime}} . \\
|\vec{P}, \sqrt{s}, J, L, S, m\rangle
\end{gathered}
$$

here $\vec{P}=\vec{p}_{1}+\vec{p}_{2}, \sqrt{s}$ - invariant mass of the system of two free particles, $L$ - orbital system center inertia (SCI), $S$ is the total spin in the SCI.

State vector of (2.4) normalized as follows:

$$
\left\langle\vec{P}, \sqrt{s}, J, L, S, m \mid \vec{P}^{\prime}, \sqrt{s^{\prime}}, J, L^{\prime}, S^{\prime}, m^{\prime}\right\rangle=N \delta\left(\vec{P}-\vec{P}^{\prime}\right) \delta\left(\sqrt{s}-\sqrt{s^{\prime}}\right) \delta_{m m} \delta_{L L^{\prime}} \delta_{S S^{\prime}}
$$

where $N$ is the standardization constant, the explicit form of which not used.

Bases of (2.2) and (2.4) linked decomposition Clebsch-Gordan coefficients for the Poincare group:

$$
\begin{aligned}
& |\vec{P}, \sqrt{s}, J, L, S, m\rangle=\sum_{m_{1}, m_{2}} \int \frac{d^{3} \vec{p}_{1}}{2 p_{10}} \int \frac{d^{3} \overrightarrow{p_{2}}}{2 p_{20}}\left|\vec{p}_{1}, m_{1} ; \vec{p}_{2}, m_{2}\right\rangle \\
& \cdot\left\langle\vec{p}_{1}, m_{1} ; \vec{p}_{2}, m_{2} \mid \vec{P}, \sqrt{s}, J, L, S, m\right\rangle
\end{aligned}
$$

where

$$
\begin{aligned}
& \left\langle\vec{p}_{1}, m_{1} ; \vec{p}_{2}, m_{2} \mid \vec{P}, \sqrt{s}, J, L, S, m\right\rangle=2 \sqrt{s}\left[\lambda\left(s, M_{1}^{2}, M_{2}^{2}\right)\right]^{-1 / 2} . \\
& \cdot 2 P_{0} \delta\left(P-p_{1}-p_{2}\right) \cdot \sum_{m_{1}, \tilde{m}_{2}} D_{m_{1} \tilde{m}_{1}}^{1 / 2}\left(p_{1}, P\right) D_{m_{2} \tilde{m}_{2}}^{1 / 2}\left(p_{2}, P\right) \cdot\left\langle 1 / 2 \tilde{m}_{1} 1 / 2 \tilde{m}_{2} \mid S m_{S}\right\rangle \cdot \\
& \cdot Y_{L m_{L}}(\vartheta, \varphi)\left\langle S L m_{S} m_{L} \mid J m\right\rangle, \\
\lambda(a, b, c)= & \left(a^{2}+b^{2}+c^{2}-2(a b+a c+b c) .\right.
\end{aligned}
$$

In the expression (2.7) expansion in spherical harmonics and the summation over the angular moments is in the SCI and the result then "shifts" in an arbitrary system through $D$-functions [17].

To build the matrix element of the electromagnetic current there is a procedure of canonical parametrization [17]. The matrix element of the current represents the sum of items, each of which is a product of the covariant and invariant States. Covariant part of such representations the matrix element describes his transformation (geometric) properties, and all dynamic information about transition described by the data of the current operator, is contained in invariant section - the matrix elements or the form. As an illustration of this technique, consider the first, the parametrization of the matrix element of the current of a diagonal on the full angular moment of [15]. 
Lorentz-covariant properties of the matrix element will be described three 4-vectors and one pseudo vector:

$$
K_{\mu}^{\prime}=\left(P+P^{\prime}\right)_{\mu}, \quad K_{\mu}=\left(P-P^{\prime}\right)_{\mu}, \quad R_{\mu}=\varepsilon_{\mu \nu \lambda \rho} P^{v} P^{\lambda} \Gamma^{\rho}\left(P^{\prime}\right), \quad \Gamma^{\mu}\left(P^{\prime}\right),
$$

$\varepsilon_{\mu \nu \lambda \rho}$ - antisymmetric tensor of the fourth rank, $\Gamma^{\mu}\left(P^{\prime}\right)$ - operator relativistic spin [15]:

$$
\Gamma^{0}\left(P^{\prime}\right)=\left(\vec{P}^{\prime} \vec{J}^{\prime}\right), \quad \vec{\Gamma}\left(P^{\prime}\right)=\sqrt{s} \vec{J}^{\prime}+\frac{\vec{P}^{\prime}\left(\vec{P}^{\prime} \vec{J}^{\prime}\right)}{P_{0}^{\prime}+\sqrt{s^{\prime}}}, \quad \Gamma^{2}=-\sqrt{s^{\prime}} J^{2}(J+1) .
$$

Linearly independent matrices is projected full moment the number of movements in initial and final States are built from vectors $P_{\mu}$ and $\Gamma\left(P^{\prime}\right)_{\mu}$ :

$$
D^{J}\left(P, P^{\prime}\right)\left(P_{\mu} \Gamma^{\mu}\left(P^{\prime}\right)\right)^{n}, \quad n=0,1,2 \ldots, 2 J .
$$

When additional conditions conservation parity, save current as well as the mutual orthogonality vectors, parametrization of the matrix element takes the following form:

$$
\begin{gathered}
\left\langle\vec{P}, \sqrt{s}, J, L, S, m\left|j_{\mu}^{0}(0)\right| \vec{P}^{\prime}, \sqrt{s^{\prime}}, J, L^{\prime}, S^{\prime}, m^{\prime}\right\rangle= \\
=\sum_{m} D_{m, m}^{J}\left\langle m^{\prime \prime}\left|\sum_{k=1}^{3}\left\{F_{k}^{L L^{\prime} S S^{\prime}} A_{k}^{\mu}\left(s, Q^{2}, s^{\prime}\right)\right\}_{+}\right| m^{\prime}\right\rangle, \\
A_{\mu}^{1}=\frac{1}{Q^{2}}\left[\left(s-s^{\prime}+Q^{2}\right) P_{\mu}+\left(s^{\prime}-s+Q^{2}\right) P_{\mu}^{\prime}\right] \\
A_{\mu}^{2}=\frac{1}{\sqrt{s^{\prime}}}\left\{\Gamma_{\mu}\left(P^{\prime}\right)-\frac{1}{2 \sqrt{s}}\left[\left(\sqrt{s}+\sqrt{s^{\prime}}\right) \frac{K_{\mu}}{Q^{2}}+\frac{\sqrt{s^{\prime}} P_{\mu}+\sqrt{s} P_{\mu}^{\prime}}{P P^{\prime}+\sqrt{s s^{\prime}}}+\frac{\sqrt{s}-\sqrt{s^{\prime}}}{\lambda\left(s, Q^{2}, s^{\prime}\right)}\left[\left(\sqrt{s}+\sqrt{s^{\prime}}\right)^{2}+Q^{2}\right] A_{\mu}^{1}\right]\left(P_{\lambda} \Gamma^{\lambda}\left(P^{\prime}\right)\right)\right\}, \\
A_{\mu}^{3}=\frac{i}{s^{\prime}} R_{\mu} .
\end{gathered}
$$

Note that in the expression (2.10) the matrix elements of the invariant form factors depend on additional invariant values of $L, L^{\prime}, S, S^{\prime}$ - invariant parameters of the degeneration of the problem (2.4):

$$
F_{k}^{L, L^{\prime}, S, S^{\prime}}=\sum_{n=0}^{2 J} f_{k n}^{L, L^{\prime}, S, S^{\prime}}\left(s, Q^{2}, s^{\prime}\right)\left(i P_{\mu} \Gamma^{\mu}\left(P^{\prime}\right)\right)^{n} .
$$

Consider now the parametrization of the matrix element of the current, non-digional full angular moment:

$$
\left\langle\vec{P}, \sqrt{s}, J, L, S, m\left|j_{\mu}^{0}(0)\right| \vec{P}^{\prime}, \sqrt{s^{\prime}}, J^{\prime} L^{\prime}, S^{\prime}, m^{\prime}\right\rangle,
$$

In the matrix element (2.12) own moments of $J$ and $J^{\prime}$ different that excludes the possibility of constructing operator spin relativistic $\Gamma_{\mu}$ (diagonal on the back) and, thus, using the technique discussed above.

For the invariant parametrization of the matrix element of (2.12) make the Lorentz transformation from the initial (laboratory) system coordinates in breit system (BS):

$$
\tilde{P}=\left(\tilde{P}_{0}, \vec{q}\right), \quad \tilde{P}^{\prime}=\left(\tilde{P}_{0}^{\prime},-\vec{q}\right), \quad \vec{q}=(0,0, q), \quad K_{\mu}^{\prime}=\left(\sqrt{K^{\prime 2}}, 0,0,0\right),
$$


$\vec{q}$ - dimensional vector, module which has the following form:

$$
q=\sqrt{\lambda\left(M_{1}^{2}, M_{2}^{2}, Q^{2}\right) /\left[8\left(M_{1}^{2}+M_{2}^{2}\right)+4 Q^{2}\right]} .
$$

In case of equality of vectors $P_{\mu}$ and $P_{\mu}^{\prime}(\mathrm{BS})$ enters (SCI):

$$
\begin{aligned}
& \left\langle\vec{P}, \sqrt{s}, J, L, S, m\left|j_{\mu}^{0}(0)\right| \vec{P}^{\prime}, \sqrt{s^{\prime}}, J^{\prime} L^{\prime}, S^{\prime}, m^{\prime}\right\rangle=\sum_{\tilde{m}, \tilde{m}^{\prime}} D_{m, \tilde{m}}^{J}(P, w) D_{m, \tilde{m}^{\prime}}^{* J^{\prime}}\left(P^{\prime}, w\right) \cdot \\
& \cdot\left\langle\overrightarrow{\tilde{P}}, \sqrt{s}, J, L, S, \tilde{m}\left|j_{\mu}^{0}(0)\right| \overrightarrow{\tilde{P}}^{\prime}, \sqrt{s^{\prime}}, J^{\prime} L^{\prime}, S^{\prime}, \tilde{m^{\prime}}\right\rangle
\end{aligned}
$$

where $w_{\mu}=K_{\mu} / \sqrt{K^{2}}$ is a 4-speed corresponding to the specified transform Lorentz.

Feasible standard transition to the canonical basis [17]:

$$
\begin{gathered}
j_{\delta}(0)=a_{\delta \sigma} \tilde{j}_{\sigma}^{1}(0), \quad \delta=1,2,3 ; \quad \sigma=1,2,3 . \\
a_{\delta \sigma}=\sqrt{\frac{2 \pi}{3}}\left(\begin{array}{ccc}
-1 & 0 & 1 \\
\mathrm{i} & 0 & \mathrm{i} \\
0 & \sqrt{2} & 0
\end{array}\right)
\end{gathered}
$$

Full parametrization of the matrix element of (2.12) looks the following follows:

$$
\begin{aligned}
& \left\langle\vec{P}, \sqrt{s}, J, L, S, m\left|\tilde{j_{0}}(0)\right| \vec{P}^{\prime}, \sqrt{s^{\prime}}, J^{\prime} L^{\prime}, S^{\prime}, m^{\prime}\right\rangle=\sum_{\tilde{m}^{\prime}, \tilde{m}, v^{\prime}, \lambda^{\prime}} D_{m \tilde{m}}^{J}(P, w) D_{m^{\prime} \tilde{m}^{\prime}}^{J^{\prime}}(P, w) \cdot \\
& \cdot\left\langle J^{\prime} \tilde{m}^{\prime} v^{\prime} \lambda^{\prime} \mid J \tilde{m}\right\rangle \cdot Y_{v^{\prime} \lambda^{\prime}}(\vartheta, \varphi) \cdot G_{J J^{\prime}}^{0, v^{\prime}}\left(s, s^{\prime}, Q^{2}\right), \\
& \left\langle\vec{P}, \sqrt{s}, J, L, S, m\left|\tilde{j}_{\sigma}^{1}(0)\right| \vec{P}^{\prime}, \sqrt{s^{\prime}}, J^{\prime} L^{\prime}, S^{\prime}, m^{\prime}\right\rangle=\sum_{\mu, \tilde{m}^{\prime}, \tilde{m}, \sigma, \lambda, \rho} D_{m \tilde{m}}^{J}(P, w) D_{m^{\prime} \tilde{m}^{\prime}}^{J^{\prime}}(P, w) \cdot \\
& \cdot\left\langle J^{\prime} \tilde{m}^{\prime} \sigma \rho \mid J \tilde{m}\right\rangle \cdot\langle 1 \sigma v \lambda \mid \mu \rho\rangle \cdot Y_{v, \lambda}(\vartheta, \varphi) \cdot G_{J J^{\prime}}^{1, v, \mu}\left(s, s^{\prime}, Q^{2}\right) .
\end{aligned}
$$

Note that the zero component of the transformed vector $j_{\mu}(0)$ is the four-dimensional scalar and three-dimensional the components of the vector form, sitting on the momentum $K^{\prime}$.

Now let's apply this procedure non-digional parameterization [17] for a description of radiation transition $\rho \rightarrow \pi \gamma$. In the composite quark model of $\pi$ and $\rho$ meson seems related condition $u$ and $\bar{d}$ with quantum numbers of quarks $J=L=S=0$ for peony and $J=S=1, L=0$ for $\rho$ meson respectively. We assume that the masses of the quarks are the same $M_{u}=M_{\bar{d}}=M$, then the matrix element of the operator the electromagnetic current of the free two-partial system data quantum numbers will be written as:

$$
\begin{aligned}
& \left\langle\vec{P}, \sqrt{s}\left|j_{\mu}^{0}(0)\right| \vec{P}^{\prime}, \sqrt{s^{\prime}}, 1,0,1, m^{\prime}\right\rangle=\int \frac{d^{3} \vec{p}_{1}}{2 p_{10}} \int \frac{d^{3} \vec{p}_{2}}{2 p_{20}} \int \frac{d^{3} \vec{p}_{1}{ }^{\prime}}{2 p_{10}^{\prime}} \int \frac{d^{3} \vec{p}_{2}{ }^{\prime}}{2 p_{20}^{\prime}} . \\
& \cdot\left\langle\vec{P}, \sqrt{s} \mid \vec{p}_{1}, m_{1} ; \vec{p}_{2}, m_{2}\right\rangle \cdot\left\langle\vec{p}_{1}, m_{1} ; \vec{p}_{2}, m_{2}\left|j_{\mu}^{0}(0)\right| \vec{p}_{1}{ }^{\prime}, m_{1}^{\prime} ; \vec{p}_{2}{ }^{\prime}, m_{2}^{\prime}\right\rangle \cdot \\
& \cdot\left\langle\vec{p}_{1}{ }^{\prime}, m_{1}^{\prime} ; \vec{p}_{2}{ }^{\prime}, m_{2}{ }^{\prime} \mid \vec{P}^{\prime}, \sqrt{s^{\prime}}, 1,0,1, m^{\prime}\right\rangle
\end{aligned}
$$


where

$$
\begin{aligned}
& \left\langle\vec{p}_{1}, m_{1} ; \vec{p}_{2}, m_{2}\left|j_{\mu}^{0}(0)\right| \vec{p}_{1}{ }^{\prime}, m_{1}^{\prime} ; \vec{p}_{2}{ }^{\prime}, m_{2}^{\prime}\right\rangle=\left\langle\vec{p}_{1}, m_{1}\left|j_{\mu-1}^{0}(0)\right| \vec{p}_{1}{ }^{\prime}, m_{1}^{\prime}\right\rangle \\
& \cdot \delta\left(\vec{p}_{2}-\vec{p}_{2}{ }^{\prime}\right) \cdot \delta_{m_{2} m_{2^{\prime}}}+\left\langle\vec{p}_{2}, m_{2}\left|j_{\mu 2}^{0}(0)\right| \vec{p}_{2}{ }^{\prime}, m_{2}^{\prime}\right\rangle \cdot \delta\left(\vec{p}_{1}-\vec{p}_{1}{ }^{\prime}\right) \cdot \delta_{m_{1} m_{1^{\prime}}}
\end{aligned}
$$

Using the parametrization [13], we obtain the expression for the single-particle matrix element of the current:

$$
\left\langle\vec{p}_{1}, m_{1}\left|j_{\mu 1}^{0}(0)\right| \vec{p}_{1}{ }^{\prime}, m_{1}^{\prime}\right\rangle=\sum_{m_{1}^{\prime \prime}} D_{m_{1} m_{1}^{\prime \prime}}^{1 / 2}\left(R\left(\vec{p}_{1}\right)\right)\left\langle m_{1}{ }^{\prime \prime}\left|\left[f_{10}\left(Q^{2}\right) K_{\mu}+i f_{30}\left(Q^{2}\right) R_{\mu}\right]\right| m_{1}^{\prime}\right\rangle
$$

here $f_{10}\left(Q^{2}\right)$ and $f_{30}\left(Q^{2}\right)$ have a sense of electrical and magnetic particle formfactors, respectively, $K_{\mu}=p_{\mu}+p_{\mu}^{\prime}, \quad R_{\mu}=\varepsilon_{\mu \nu \lambda \rho} p_{1}^{v} p_{1}^{\prime},{ }^{\lambda} \Gamma^{\rho}\left(p_{1}^{\prime}\right)$. Clebsch-Gordan coefficients are defined as follows:

$$
\begin{aligned}
& \left\langle\vec{P}, \sqrt{s} \mid \vec{p}_{1}, m_{1} ; \vec{p}_{2}, m_{2}\right\rangle=2 \sqrt{s} \frac{1}{\sqrt{4 \pi}}\left[\lambda\left(s, M^{2}, M^{2}\right)\right]^{-1 / 2} \\
& \cdot 2 P_{0} \delta\left(P-p_{1}-p_{2}\right) \cdot \sum_{\tilde{m}_{1} \tilde{m}_{2}} D_{m_{1} \tilde{m}_{1}}^{1 / 2}\left(R\left(\vec{p}_{1}\right)\right) D_{m_{2} \tilde{m}_{2}}^{1 / 2}\left(R\left(\vec{p}_{2}\right)\right) \cdot\left\langle 1 / 2 \tilde{m}_{1} 1 / 2 \tilde{m}_{2} \mid 00\right\rangle \\
& \left\langle\vec{P}^{\prime}, \sqrt{s}, 1,0,1, m^{\prime} \mid \vec{p}_{1}^{\prime}, m_{1}^{\prime} ; \vec{p}_{2}^{\prime}, m_{2}^{\prime}\right\rangle=2 \sqrt{s} \frac{1}{\sqrt{4 \pi}}\left[\lambda\left(s^{\prime}, M^{2}, M^{2}\right)\right]^{-1 / 2} \\
& \cdot 2 P_{0}^{\prime} \delta\left(P^{\prime}-p_{1}^{\prime}-p_{2}^{\prime}\right) \cdot \sum_{\tilde{m}_{1}^{\prime} \tilde{m}_{2}^{\prime}} D_{m_{1}^{\prime} \tilde{m}_{1}^{\prime}}^{1 / 2}\left(R\left(\vec{p}_{1}^{\prime}\right)\right) D_{m_{2}^{\prime} \tilde{m}_{2}^{\prime}}^{1 / 2}\left(R\left(\vec{p}_{2}^{\prime}\right)\right) \cdot\left\langle 1 / 2 \tilde{m}_{1}{ }^{\prime} 1 / 2 \tilde{m}_{2}{ }^{\prime} \mid 1 m_{S}^{\prime}\right\rangle \cdot \\
& \cdot\left\langle 1 m_{S}^{\prime} 00 \mid 1 m^{\prime}\right\rangle .
\end{aligned}
$$

Using non-digional parameterization [17] matrix item current in the basis of (2.4) can be represented as:

$$
\begin{aligned}
& \left\langle\vec{P}, \sqrt{s}|\tilde{j} 0(0)| \vec{P}^{\prime}, \sqrt{s^{\prime}}, 1,0,1, m^{\prime}\right\rangle=\sum_{\tilde{m}^{\prime}, \tilde{m}, v^{\prime}, \lambda^{\prime}} D_{0 \tilde{m}}^{0}(P, w) D_{m^{\prime} \tilde{m}^{\prime}}^{1}\left(P^{\prime}, w\right) \cdot \\
& \cdot\left\langle 1 \tilde{m}^{\prime} v^{\prime} \lambda^{\prime} \mid 0 \tilde{m}\right\rangle \cdot Y_{v^{\prime} \lambda^{\prime}}(\vartheta, \varphi) \cdot G_{01}^{0, v^{\prime}}\left(s, s^{\prime}, Q^{2}\right), \\
& \left\langle\vec{P}, \sqrt{s}\left|\tilde{j}_{\sigma}^{1}(0)\right| \vec{P}^{\prime}, \sqrt{s^{\prime}}, 1,0,1, m^{\prime}\right\rangle=\sum_{\mu, \tilde{m}^{\prime}, \tilde{m}, \sigma, \lambda, \rho} D_{0 \tilde{m}}^{0}(P, w) D_{m^{\prime} \tilde{m}^{\prime}}^{1}\left(P^{\prime}, w\right) \cdot \\
& \cdot\left\langle 1 \tilde{m}^{\prime} \sigma \rho \mid 0 \tilde{m}\right\rangle \cdot\langle 1 \sigma v \lambda \mid \mu \rho\rangle \cdot Y_{v \lambda}(\vartheta, \varphi) \cdot G_{01}^{1, v, \mu}\left(s, s^{\prime}, Q^{2}\right) .
\end{aligned}
$$

Equating expressions (2.19) and (2.24) - (2.25) with the account of (2.15)-(2.16) and (2.20)(2.21)by integration in the system $\vec{P}^{\prime}=0, \vec{P}=(0,0, P)$, we obtain analytical expressions for the free two-particle formfactors.

$$
G_{01}^{111}\left(s, Q^{2}, s^{\prime}\right)=\frac{\sqrt{2} \cdot \Theta\left(s, Q^{2}, s^{\prime}\right)\left(s+s^{\prime}+Q^{2}\right)^{2}}{2 \sqrt{s-4 M^{2}} \sqrt{s^{\prime}-4 M^{2}} \sqrt{4 M^{2}+Q^{2}}\left[\lambda\left(s,-Q^{2}, s^{\prime}\right)\right]^{1 / 2}} .
$$




$$
\begin{aligned}
& \cdot \cos \left[\left(\omega_{1}+\omega_{2}\right) / 2\right]\left[\frac{s^{\prime}\left(s^{\prime}-s+3 Q^{2}\right)}{\left[\lambda\left(s,-Q^{2}, s^{\prime}\right)\right]^{1 / 2}}\left(G_{M}^{u}\left(Q^{2}\right)+G_{M}^{\bar{d}}\right)\left(Q^{2}\right)\right]+ \\
& +\sin \left[\left(\omega_{1}+\omega_{2}\right) / 2\right]\left[\frac{\left(s^{\prime}-s-Q^{2}\right)}{\left(s+s^{\prime}+Q^{2}\right)} \frac{\xi\left(s, s^{\prime}, Q^{2}\right)}{\sqrt{s^{\prime}}}\left(G_{M}^{u}\left(Q^{2}\right)+G_{M}^{\bar{d}}\left(Q^{2}\right)\right)\right]- \\
& -\sin \left[\left(\omega_{1}+\omega_{2}\right) / 2\right]\left[\xi\left(s, s^{\prime}, Q^{2}\right) \frac{4 M}{\left(s+s^{\prime}+Q^{2}\right)}\left(G_{E}^{u}\left(Q^{2}\right)+G_{E}^{\bar{d}}\left(Q^{2}\right)\right)\right], \\
& G_{01}^{01}\left(s, Q^{2}, s^{\prime}\right)=\frac{\sqrt{2} \cdot \sqrt{\pi} \Theta\left(s, Q^{2}, s^{\prime}\right)\left(s+s^{\prime}+Q^{2}\right)}{2 \sqrt{s-4 M^{2}} \sqrt{s^{\prime}-4 M^{2}} \sqrt{4 M^{2}+Q^{2}}\left[\lambda\left(s,-Q^{2}, s^{\prime}\right)\right]^{1 / 2}} . \\
& \cdot \cos \left[\left(\omega_{1}+\omega_{2}\right) / 2\right]\left[M \cdot\left[\lambda\left(s,-Q^{2}, s^{\prime}\right)\right]^{1 / 2}\left(G_{E}^{u}\left(Q^{2}\right)+G_{E}^{\bar{d}}\right)\left(Q^{2}\right)\right]+ \\
& +\sin \left[\left(\omega_{1}+\omega_{2}\right) / 2\right]\left[\frac{\xi\left(s, s^{\prime}, Q^{2}\right) \lambda\left(s,-Q^{2}, s^{\prime}\right)}{s^{\prime}\left(s+s^{\prime}+Q^{2}\right)}\left(G_{M}^{u}\left(Q^{2}\right)+G_{M}^{\bar{d}}\left(Q^{2}\right)\right],\right. \\
& G_{01}^{121}\left(s, Q^{2}, s^{\prime}\right)=\frac{\sqrt{2} \cdot \Theta\left(s, Q^{2}, s^{\prime}\right)\left(s+s^{\prime}+Q^{2}\right)}{2 \sqrt{s-4 M^{2}} \sqrt{s^{\prime}-4 M^{2}} \sqrt{4 M^{2}+Q^{2}}\left[\lambda\left(s,-Q^{2}, s^{\prime}\right)\right]^{1 / 2}} . \\
& \cdot \sin \left[\left(\omega_{1}+\omega_{2}\right) / 2\right]\left[\frac{\sqrt{-2 s^{\prime}\left(8 M^{2}-s+Q^{2}+\left(s+Q^{2}\right)^{2}+s^{\prime 2}\right)} 3\left[\lambda\left(s,-Q^{2}, s^{\prime}\right)\right]^{1 / 2}}{8 s^{\prime}}\right] \\
& \cdot\left(G_{M}^{u}\left(Q^{2}\right)+G_{M}^{\bar{d}}\right)\left(Q^{2}\right)+ \\
& +\sin \left[\left(\omega_{1}+\omega_{2}\right) / 2\right]\left[M \frac{\xi\left(s, s^{\prime}, Q^{2}\right)}{s+s^{\prime}+Q^{2}}\left(G_{M}^{u}\left(Q^{2}\right)+G_{M}^{\bar{d}}\left(Q^{2}\right)\right)\right]+ \\
& +\cos \left[\left(\omega_{1}+\omega_{2}\right) / 2\right]\left[\frac{\left[\left(s+Q^{2}\right)^{2}-s^{\prime 2}\right] \cdot\left(s+s^{\prime}+Q^{2}\right)+\left[\lambda\left(s,-Q^{2}, s^{\prime}\right)\right]^{1 / 2}}{\left(s+s^{\prime}+Q^{2}\right)}\right] \\
& \cdot\left(G_{M}^{u}\left(Q^{2}\right)+G_{M}^{\bar{d}}\left(Q^{2}\right)\right)+ \\
& +\cos \left[\left(\omega_{1}+\omega_{2}\right) / 2\right]\left[\frac{M \cdot\left[\left(s+Q^{2}\right)^{2}-s^{\prime 2}\right]}{\sqrt{s^{\prime}}\left[\lambda\left(s,-Q^{2}, s^{\prime}\right)\right]^{1 / 2}} \cdot\left(G_{E}^{u}\left(Q^{2}\right)+G_{E}^{\bar{d}}\left(Q^{2}\right)\right)\right], \\
& G_{01}^{101}\left(s, Q^{2}, s^{\prime}\right)=\frac{\Theta\left(s, Q^{2}, s^{\prime}\right)\left(s+s^{\prime}+Q^{2}\right)}{2 \sqrt{s-4 M^{2}} \sqrt{s^{\prime}-4 M^{2}} \sqrt{4 M^{2}+Q^{2}}\left[\lambda\left(s,-Q^{2}, s^{\prime}\right)\right]^{1 / 2}} . \\
& \cdot \sin \left[\left(\omega_{1}+\omega_{2}\right) / 2\right]\left[M \frac{\xi\left(s, s^{\prime}, Q^{2}\right)}{s+s^{\prime}+Q^{2}}\left(G_{E}^{u}\left(Q^{2}\right)+G_{E}^{\bar{d}}\left(Q^{2}\right)\right)\right]- \\
& -\cos \left[\left(\omega_{1}+\omega_{2}\right) / 2\right]\left[\frac{\left[\left(s+Q^{2}\right)^{2}-s^{\prime 2}\right] \cdot\left(s+s^{\prime}+Q^{2}\right)+\left[\lambda\left(s,-Q^{2}, s^{\prime}\right)\right]^{1 / 2}}{\left(s+s^{\prime}+Q^{2}\right)}\right] \\
& \cdot\left(G_{M}^{u}\left(Q^{2}\right)+G_{M}^{\bar{d}}\left(Q^{2}\right)\right)- \\
& -\cos \left[\left(\omega_{1}+\omega_{2}\right) / 2\right]\left[\frac{M \cdot\left[\left(s+Q^{2}\right)^{2}-s^{\prime 2}\right]}{\sqrt{s^{\prime}}\left[\lambda\left(s,-Q^{2}, s^{\prime}\right)\right]^{1 / 2}} \cdot\left(G_{E}^{u}\left(Q^{2}\right)+G_{E}^{\bar{d}}\left(Q^{2}\right)\right)\right],
\end{aligned}
$$


where:

$$
\begin{gathered}
\Theta\left(s, Q^{2}, s^{\prime}\right)=\vartheta\left(s^{\prime}-s_{1}\right)-\vartheta\left(s^{\prime}-s_{2}\right), \\
s_{1,2}=2 M^{2}+\frac{1}{2 M^{2}}\left(2 M^{2}+Q^{2}\right)\left(s-2 M^{2}\right) \mp \frac{1}{2 M^{2}} \sqrt{Q^{2}\left(Q^{2}+4 M^{2}\right) s\left(s-4 M^{2}\right)},
\end{gathered}
$$

$\vartheta$ is a step function.

$$
\begin{gathered}
\xi\left(s, s^{\prime}, Q^{2}\right)=\sqrt{s s^{\prime} Q^{2}-M^{2} \lambda\left(s, Q^{2}, s^{\prime}\right)}, \\
\omega_{1}=\arctan \frac{\xi\left(s, s^{\prime}, Q^{2}\right)}{M\left[\left(\sqrt{s}+\sqrt{s^{\prime}}\right)^{2}+Q^{2}\right]+\sqrt{s s^{\prime}}\left(\sqrt{s}+\sqrt{s^{\prime}}\right)}, \\
\omega_{2}=\arctan \frac{\xi\left(s, s^{\prime}, Q^{2}\right)\left(2 M+\sqrt{s}+\sqrt{s^{\prime}}\right)}{M\left(s+s^{\prime}+Q^{2}\right)\left(2 M+\sqrt{s}+\sqrt{s^{\prime}}\right)+\sqrt{s s^{\prime}}\left(4 M^{2}+Q^{2}\right)},
\end{gathered}
$$

$G_{M}\left(Q^{2}\right)$ and $\left(G_{E}\left(Q^{2}\right)\right.$ - magnetic and electric form factors, which are expressed as single-particle following [15]:

$$
\begin{gathered}
f_{10}\left(Q^{2}\right)=\frac{2 M}{\sqrt{4 M^{2}+Q^{2}}} G_{E}\left(Q^{2}\right), \quad f_{30}\left(Q^{2}\right)=-\frac{4}{M \sqrt{4 M^{2}+Q^{2}}} G_{M}\left(Q^{2}\right), \\
G_{E}\left(Q^{2}\right)=e_{q} f_{q}\left(Q^{2}\right), \quad G_{M}\left(Q^{2}\right)=\left(e_{q}+k_{q}\right) f_{q}\left(Q^{2}\right),
\end{gathered}
$$

where $e_{q}$ is the charge of the quark mass, $k_{q}$ - anomalous magnetic moment quark in natural units.

\section{References}

[1] Arnaldi R [NA60 Collaboration]. Phys.Lett. 2009. V B667. P. 260.

[2] Usai G [NA60 Collaboration]. Nucl.Phys. 2011. V A855. P. 189.

[3] Uras A [NA60 Collaboration]. Phys.Conf.Ser. 2011. V 270. N 012038. P.1.

[4] Archilli F [KLOE-2 Collaboration]. arxiv:1110.0411.2011. P. 12.

[5] Amsler C. Phys. Lett B. 2008. V 667. P. 1.

[6] Sakurai J.J. Phys.Lett. 1969. V 22. P.981.

[7] Braun V. Phys.Lett B. 1994. V 328. P.457.

[8] Cardarelli F., Grach I.L., Narodetskii I.M. Phys. Lett B. 1995. V 359. P.1.

[9] Cross F. Phys.Rev. Lett. 1993. V 71. P.2555.

[10] Maris P., Tandy P.C. Phys. Rev C. 2002. V 65. N 045211. P. 13.

[11] Ivashyn S.A. Nucl.Phys.Inv. 2012. V 57. P. 179.

[12] Dirac, P.A.M. Rev.Mod.Phys. 1949. V 21. P. 399.

[13] Krutov A.F., Troitsky V.E., FECHAI. 2009. V. 40. P. 269.

[14] Krutov A.F., Troitsky V.E., Phys. Rev C. 2002. V. 65. P. 045501.

[15] Krutov A.F., Troitsky V.E., Phys. Rev C. 2003. V. 68. P. 018501.

[16] Krutov A.F., Troitsky V.E., Tsirova N.A. Phys.A. 2008. V. 41. P. 255401.

[17] Cheshkov A.A., Shirokov U.M. ZHETF. 1963. V. 44. P. 1983. 